\title{
Interprofessional Health Sciences Education: It's Time to Overcome Barriers and Excuses
}

\author{
Michael Wilkes, MD, $P h D^{7}$ and Robin Kennedy, PhD, MSW \\ ${ }^{1}$ School of Medicine, University of California, Davis, CA, USA; 22Sacramento State University, Sacramento, CA, USA.
}

J Gen Intern Med 32(8):858-9

DOI: $10.1007 / \mathrm{s} 11606-017-4069-\mathrm{z}$

(c) Society of General Internal Medicine 2017

P hysicians and medical educators have been slow to recognize the practice of medicine as a team sport. Over the past decade there have been dramatic changes in American hospitals, health care systems, and how health care is organized which have affected the way we deliver health care, the role of the health care professional, and the expectations of the patient. These changes have, in turn, impacted how the health care team functions and interacts. Interprofessional care has become the norm, with the physician being but one important player on the team. For too long the division between physicians and other health care providers has been hierarchical and rigid, with physicians feeling they were in charge and with other occupations seen as subordinate. We have done little to help our trainees understand the role and approaches to care offered by other team members, which includes social workers, psychologists, nurses, and physician assistants/ nurse practitioners, as well as complementary and alternative care providers including chiropractors, acupuncturists, and others whom our patients visit for care. When we attempt to teach "systems-based practice," too often the model has been to teach our trainees leadership skills with the explicit and implicit assumptions that they will always be captains [of the ship] rather than just one important member of the crew.

In this issue of JGIM, Alexandraki et al. detail the institutional and professional resistance to change within the culture of both medicine and academia. ${ }^{1}$ Despite the Liaison Committee on Medical Education (LCME) mandate to include interprofessional practice within professional health care training, most doctors, nurses, social workers, physical therapists, and others are still learning their trade in separate silos. In medicine, this is a result of our medical training systems having evolved from a Flexnerian academic mind-set rather than from a health care delivery mind-set. When we place trainees in academic hospitals, they are typically organized in silos and units, taught by one specialty at a time and focused on disease management rather than on broader issues of health. Medical education programs would never fail to teach our trainees cardiac auscultation, history taking, or anatomy, because these are considered by most in the profession as

Published online May 11, 2017 mission-critical. In stark contrast, the skills and attitudes involved in interprofessional education (IPE) are often considered fluff.

While excuses for excluding interprofessional experiences from the curriculum have been heard for at least a decade, Alexandraki et al. have given us some indication that the attitudes of internal medicine (IM) clerkship directors are starting to change. Slowly, schools have taken small steps to incorporate IPE into the curriculum, although the additions often seem perfunctory. While $71 \%$ of respondents believed that IPE should be part of IM clerkships, in practice, respondents indicated that their programs often front-loaded their IPE experiences prior to their clerkship year, with decreasing IP exposure in the clinical years of medical school, when it would arguably have the greatest impact.

Most programs have included IPE in their curricula by placing groups of students from different professions together in lectures or including them on ward rounds. More intentional and integrated IPE models include students from two or three disciplines addressing a clinical case presented as text, using a simulation lab, or using standardized patients, while students observe others conducting their discipline-specific assessments. Given that IPE is rarely more than an occasional addition within long-established medical and nursing curricula, most of the current models of IPE not only lack a diversity of health care disciplines, but also lack the time needed to explore and understand cultural differences in the approach to care.

Alexandraki et al.'s concluding remarks highlight the need for IPE curriculum and faculty development and other "clinical immersion experiences." What is still missing from IPE curriculum is the core ingredient of interprofessional teams in practice: relationships. Students gain a deeper understanding of health and healing when they learn from, and with, other health care students. Simply increasing IPE clerkship experiences or providing faculty development for IM faculty will not necessarily make a student "interprofessional teamready". What is needed is for trainees to have the time and opportunity to share their passion for medicine, patient care, and approaches to health care. Relationship building does not happen when individuals are briefly exposed to one another in the hall or at the bedside to listen to each other's professional assessment. Relationship-based IPE requires repeated longitudinal interactions that include time to get to know one another.

Difficult interactions such as interprofessional conflicts also offer unique learning opportunities. Conflict refers to a 
struggle between people with opposing needs, ideas, values, or goals, and it is inevitable when people work together. Nowhere is this truer than in health care, where the overall focus is often on the patient, but our perspectives, experiences, personal styles, gender, race, religion, and a host of other factors color our interactions. If not managed properly, conflict can have negative effects on patient care, job satisfaction, and professional productivity. ${ }^{2}$ Eighty percent of health care workers have reported that they experienced intimidation and conflict with other members of the health care team. ${ }^{3}$ The impact of conflict on patient safety and care are well documented. ${ }^{4}$ In many ways, the need for education around conflict is a metaphor for the need for IPE. Successful strategies used to resolve conflict include direct communication, willingness to explore solutions, and demonstration of respect and humility. These IPE communication skills are rarely taught in health sciences training programs.

As mentioned by Alexandraki et al., there are a host of excuses provided for why IPE has not happened (differences in work/class schedules, lack of time, workload difference, lack of faculty expertise, etc.). Not surprisingly, many of these barriers have their roots in interprofessional attitudes toward collaboration and the low priority given IPE education. ${ }^{5}$ As interprofessional teams are becoming increasingly important in health care delivery, the benefits and liabilities of interprofessional collaboration are becoming more apparent as well. Welp and colleagues ${ }^{6}$ found that interpersonal teamwork led to improved morale, decreased burnout, and increased patient safety. While there is certainly a need for new curricula emphasizing IPE in areas such as problem solving, communication skills, and conflict management, simply teaching these in a revised formal curriculum is not enough. Much has been written about the informal or hidden curriculum and its power to reinforce or extinguish previously learned behaviors. ${ }^{7}$ Nowhere is this more important than with IPE. Of course we should provide creative, innovative, interactive exercises that involve health science trainees working together in case-based learning or in such settings as hospices, senior centers, jails, and drug treatment centers, where IPE is crucial to highquality health care. However, if the role models our trainees are exposed to outside the classroom or simulation lab do not reinforce core concepts such as respect, collaboration, and careful listening to all disciplines, there is little hope of changing our trainees' behaviors.

Many of us believe that assessment is a potent driver of learning, and another important tool for changing behavior. Alexandraki et al. found little formal assessment of IPE in IM clerkships. Clearly, IPE assessments do not lend themselves to multiple-choice questions, short answers, fill-in-the-blank questions, or board examinations. Fortunately, many programs have evolved their assessment exercises to include objective structured clinical exercises (OSCEs), objective structured video exercises (OSVEs), simulation, and some variation on 360-degree evaluations, where trainees are assessed by faculty, staff, and patients/families on a host of behaviors, many of which relate to IPE. At the end of the day, the goal is to determine what trainees have learned rather than what was taught. A common educator fallacy is apparent when faculty report a long list of content that they have taught their students, but students report a far more limited list of what they actually learned.

While the LCME has attempted to elevate the importance of IPE, it remains simply one of many boxes to be checked during curriculum accreditation. There are no required outcomes. It is time for some pedagogical imagination. Interprofessional faculty development would be one place to start with an eye toward creating new learning opportunities that focus on problem solving rather than factual content. Some schools have begun sponsoring "lunch and learn" faculty development opportunities, where faculty from all health professions are invited and interprofessional activities are planned. Simply introducing faculty from differing disciplines can be the first small step toward moving outside the silo. Lastly, and most importantly, we all need to cultivate a culture change within the clinics and hospitals. Our students learn to practice as a team only when their instructors and mentors model the respect and trust for colleagues that we would like to see in the next generation of health care providers.

Corresponding Author: Michael Wilkes, MD, PhD; School of MedicineUniversity of California, Davis, CA, USA (e-mail: mwilkes@ucdavis.edu).

\section{Compliance with ethical standards:}

Conflict of interest: The authors declare that they do not have a conflict of interest.

\section{REFERENCES}

1. Alexandraki I, Hernandez CA, Torre DM, Chretien KC. Interprofessional Education in the Internal Medicine Clerkship Post-LCME Standard Issuance: Results of a National Survey. J Gen Intern Med. 2017. doi:10. 1007/s11606-017-4004-3

2. Greer L, Saygi $\mathbf{O}$, Aaldering $\mathbf{H}$, de Dreu C. Conflict in medical teams: opportunity or danger? Med Educ. 2012;46(10):935-942. doi:10.1111/j. 1365-2923.2012.04321.

3. PricewaterhouseCoopers. What works: healing the healthcare staffing shortage. New York: PricewaterhouseCoopers; 2007. Retrieved from: http: / /www.pwc.com/extweb/pwcpublications.nsf/docid/ 674D1E79A678A0428525730D006B74A9.

4. Coombs, M. Power and conflict in intensive care clinical decision making. Intensive Crit Care Nurs. 2003;19:125-135.

5. Bridges D, Davidson R, Odegard P, Maki I, Tomkowiak J. Interprofessional collaboration: three best practice models of interprofessional education. Med Educ Online. 2011;16. doi: 10.3402/meo.v16i0.6035.

6. Welp A, Meier LL, Manser T. The interplay between teamwork, clinicians' emotional exhaustion, and clinician-rated patient safety: a longitudinal study. Crit Care. 2016;20:110.

7. Wilkes M. Hidden agendas teaching and learning in medicine. In: Mitchell DE, Ream RK, eds. Professional Responsibility: The Fundamental Issue in Education and Health Care Reform. New York: Springer International Publishing; 2014:141-154 